\title{
Associations between the rs6010620 Polymorphism in RTEL1 and Risk of Glioma: a Meta-analysis of 20,711 Participants
}

\author{
Yao Wu ${ }^{1 \&}$, Xiang Tong ${ }^{1 \&}$, Ling-Li Tang ${ }^{2}$, Kai Zhou ${ }^{1}$, Chuan-Hong Zhong ${ }^{1}$, Shu Jiang1*
}

\begin{abstract}
Background: Associations between the rs6010620 polymorphism in the regulator of telomere elongation helicase1 (RTEL1) gene and glioma have been widely reported but the results were not inconclusive. The aim of the current study was to investigate the association between the rs6010620 polymorphism in RTEL1 gene and risk of glioma by meta-analysis. Materials and Methods: We searched PubMed, Embase, Wanfang Weipu and CNKI (China National Knowledge Infrastructure) databases, which included all research published 05 May 2014. A total of 8,292 cases and 12,419 controls from 14 case-control studies involving the rs6010620 polymorphism in the RTEL1 gene were included. Statistical analysis was performed using STATA 12.0 software. Results: The results indicated that the rs6010620 polymorphism in RTEL1 gene was indeed associated with risk of glioma $(\mathrm{OR}=1.474,95 \% \mathrm{CI}=1.282-1.694, p<0.001)$. On subgroup analysis by ethnicity, we found associations between the rs6010620 polymorphism in the RTEL1 gene and risk of glioma in both Caucasians and Asians. Conclusions: The current meta-analysis suggested that the rs6010620 polymorphism in the RTEL1 gene might increase risk of glioma. In future, larger case-control studies are needed to confirm our results.
\end{abstract}

Keywords: RTEL1 - polymorphism - glioma - risk - meta-analysis

Asian Pac J Cancer Prev, 15 (17), 7163-7167

\section{Introduction}

Glioma are the most common adults tumors of the central nervous system (CNS), accounting for a majority $(80 \%)$ of glioblastoma (Dolecek et al., 2012; Walsh et al., 2013). Although with optimal treatment, glioma have high mortality and morbidity, and median survival is just about 12-15 months (Ahmed et al., 2014). However, the pathogenesis of glioma remains unclearly until now. Exposure to ionizing radiation may be the only identified physical risk factor, but which accounts for few cases (Bondy et al., 2008). Besides, genetic susceptibility might take a crucial role in modifying the occurrence of glioma (Zhao et al., 2014). Previous large number of studies revealed that several candidate risk genetic variants of genes may associate with glioma, including 5p15.33 (rs2736100, TERT), 8q24.21 (rs4295627, CCDC26), 9p21.3 (rs4977756, CDKN2A-CDKN2B), 11q23.3 (rs498872, PHLDB1), and 20q13.33 (rs6010620, RTEL1) ( Shete et al., 2009; Schoemaker et al., 2010; Safaeian et al., 2013). In addition, other studies also indicated that the rs6010620 polymorphism in regulator of telomere elongation helicase1 (RTEL1) gene was associated with the risk of glioma (Chen et al., 2011; Li et al., 2013a; Walsh et al., 2013).

The human RTEL1 gene is located on chromosome 20q13.33. One of the important polymorphism in RTEL1 gene named rs6010620 which locate at intron 12. RTEL1 is a DNA helicase. It is essential for regulation of telomere length, and take an important role in maintaining telomere integrity (Vannier et al., 2014), both at sites of mitotic DNA damage and at telomeres (Ding et al., 2004; Adelman and Boulton, 2010; Youds et al., 2010 Uringa et al., 2011;). Thus, genetic variations in RTEL1 genes may influence capacity of telomere maintenance. Telomeres are specialized DNA structures, which locate at the end of chromosomes and play crucial role in stabilizing chromosomes by protecting them from endto-end fusion and DNA degradation (Blackburn et al., 2006). The destruction of telomeres impairs their role in protecting chromosome ends and ultimately results in chromosomal instability (Hackett et al., 2002). Therefore, telomere erosion may lead to two inconsistent results: tumor inhibition by causing cell death or tumor promotion by inducing genetic instability, a critical event in the occurrence of carcinogenesis. It has been reported that lacking of telomeres may also influence genome-wide DNA methylation, which may regulate oncogene and oncosuppressor gene expression. So mutations in RTEL1 gene may play a critical role in the initiation of tumor. In addition, previous studies have indicated that genetic variations in RTEL1 gene associated with the risk of liver carcinogenesis, gastrointestinal tract tumors, and breast cancers in mammals (Muleris et al., 1995; Pitti et al., 1998; 
Bai et al., 2000; Wu et al., 2012).

Many studies have revealed that the associations between the rs6010620 polymorphism in RTEL1 gene and risk of glioma, however relatively small sample size of a single study may not have enough power to detect effects of the rs6010620 polymorphism in RTEL1 gene on glioma. A previous meta-analysis has been performed by Zhao W, et al. (Zhao et al., 2014), but there are something wrong in their meta-analysis. Besides, increasing articles focus on the association between the rs6010620 polymorphism in RTEL1 gene and risk of glioma in recent years. For above reasons, we performed an update meta-analysis based on all eligible case-control studies to evaluate the association between the rs 6010620 polymorphism and risk of glioma. To our knowledge, this is the most comprehensive metaanalysis to date investigating the association between rs6010620 polymorphism in RTEL1 gene and risk of glioma which is more reliable.

\section{Materials and Methods}

\section{Searching}

Two independent reviewers carried out a publication by searching in Pubmed, Embase, CNKI, Wanfang, and Weipu databases with the following search items: 'regulator of telomere elongation helicase1' or 'RTEL1' or 'rs6010620'; 'polymorphism' or 'variant' or 'mutation'; 'glioma'. Last search was performed on 05 may 2014. Publication language was not restricted. All studies concerning the associations between the RTEL1 rs6010620 polymorphism and risk of glioma were included.

\section{Inclusion criteria}

Eligible studies included in our study have to meet the following criteria: (1) It evaluated the association between susceptibility of glioma and the rs6010620 polymorphism in RTEL1 gene in each study, (2) studies should be designed as a human beings, (3) a case-control study, (4) provide detailed genotype data for the calculation of odds ratio (OR) and 95\% confidence interval (95\%CI), (5) genotype frequencies are consistent with Hardy-Weinberg equilibrium (HWE) in control groups., (6) If the authors published more than one articles with the same case series or overlapped case series, studies with the largest sample size or the latest publication time were included. The following exclusive items: (1) family-based studies (2) studies did not show genotype frequencies or numbers in the original studies, (3) reviews and abstracts.

\section{Data extraction}

For each study, we extracted the following information: the first author's name, year of publication, country of origin, ethnicity, case age, the number of genotyped cases and controls, sample size and type of cancer.

\section{Statistical analysis}

Meta-analysis was carried out with the STATA 12.0 software. HWE was initially detected by the Person's $\chi^{2}$ test. The $\mathrm{Q}$ test was performed to examine the heterogeneity: if $p>0.05$, we chose the fixed-effects model; Otherwise, we selected the random-effects model. The strength of association was evaluated by odds ratio (OR) with the corresponding $95 \%$ confidence intervals (CI). The genetic model assessed for the pooled OR of the polymorphism was in dominant models (GG+GA vs. AA). For each analysis, other genetic models were assessed to the association with the risk of glioma (GG vs. GA+AA, GG vs. AA, GA vs. AA, and G vs. A). To analyze the ethnicity-specific effects, stratified analysis was carried out with ethnicity (European and Asian). In addition, we

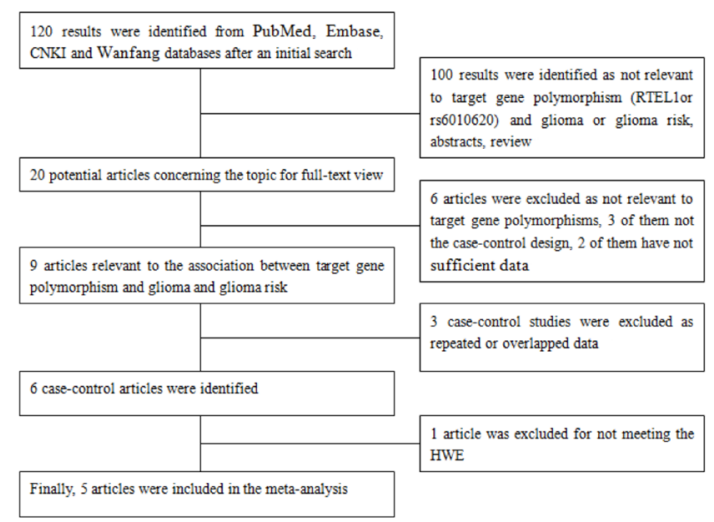

Figure 1. The Flow Diagram of Included and Excluded Studies

Table 1. Characteristics of Case-Control Studies Included in Meta-Analysis

\begin{tabular}{|c|c|c|c|c|c|c|}
\hline \multirow[t]{2}{*}{ First authors } & \multirow[t]{2}{*}{ Years } & \multirow[t]{2}{*}{ Countries } & \multirow[t]{2}{*}{ Ethnicity } & \multicolumn{2}{|c|}{ Sizes } & \multirow{2}{*}{$\begin{array}{c}\mathrm{HWE}^{(\mathrm{a})} \\
p\end{array}$} \\
\hline & & & & cases & controls & \\
\hline Walsh K M & 2013 & America & Caucasian & 2282 & 1903 & I \\
\hline $\mathrm{Li} \mathrm{G}$ & 2013 & China & Asian & 629 & 644 & 0.18 \\
\hline Safaeian M $\left(\mathrm{NCI}^{(\mathrm{b})}\right)$ & 2013 & America & Caucasian & 322 & 386 & 0.92 \\
\hline Safaeian M $\left(\mathrm{NIOSH}^{(\mathrm{c})}\right)$ & 2013 & America & Caucasian & 300 & 539 & 0.69 \\
\hline Safaeian M (PLCO $\left.{ }^{(\mathrm{d})}\right)$ & 2013 & America & Caucasian & 133 & 855 & 0.74 \\
\hline Safaeian M (ATBC ${ }^{(\mathrm{e})}$ ) & 2013 & Finland & Caucasian & 37 & 1270 & 0.15 \\
\hline Safaeian M (AHS ${ }^{(\mathrm{f}))}$ & 2013 & America & Caucasian & 18 & 35 & 0.69 \\
\hline Schoemaker MJ(Denmark) & 2010 & England & Caucasian & 122 & 147 & 0.72 \\
\hline Schoemaker MJ(Finland) & 2010 & England & Caucasian & 95 & 96 & 0.8 \\
\hline Shete (England) & 2009 & America & Caucasian & 631 & 1433 & 0.57 \\
\hline Shete (America) & 2009 & America & Caucasian & 1247 & 2235 & 0.62 \\
\hline Shete (France) & 2009 & America & Caucasian & 1332 & 1545 & 0.48 \\
\hline Shete (German) & 2009 & America & Caucasian & 499 & 557 & 0.35 \\
\hline Shete (Sweden) & 2009 & America & Caucasian & 645 & 774 & 0.07 \\
\hline
\end{tabular}

(a): Hardy-Weinberg equilibrium. (b): National Cancer Institute glioma case-control study. (c): National Institute of Occupational Safety and Health glioma case-control study. (d): glioma cases and controls identified from the Prostate, Lung, Colorectal and Ovarian Cancer Screening Trial. (e): glioma cases and controls identified from the Alpha-Tocopherol, Beta-Carotene Cancer Prevention Study. (f): glioma cases and controls identified from the Agricultural Health Study 
Table 2. Distribution of the G Genotype Among Patients with Glioma and Controls Included

\begin{tabular}{|c|c|c|c|c|c|c|c|c|c|c|}
\hline \multirow[t]{2}{*}{ First authors } & \multicolumn{5}{|c|}{ Cases } & \multicolumn{5}{|c|}{ Controls } \\
\hline & AA & $\mathrm{AG}$ & GG & A & G & AA & AG & GG & A & G \\
\hline Walsh K M & 68 & 673 & 1541 & 809 & 3755 & l & l & l & 1154 & 2652 \\
\hline $\mathrm{Li} \mathrm{G}$ & 293 & 261 & 75 & 847 & 411 & 337 & 267 & 40 & 941 & 347 \\
\hline Safaeian M (NCI $\left.{ }^{(b)}\right)$ & 11 & 93 & 218 & 115 & 529 & 20 & 134 & 231 & 174 & 596 \\
\hline Safaeian M (NIOSH $\left.{ }^{(c)}\right)$ & 12 & 106 & 182 & 130 & 470 & 25 & 175 & 339 & 225 & 853 \\
\hline Safaeian M (PLCO $\left.{ }^{(\mathrm{d})}\right)$ & 0 & 29 & 104 & 29 & 237 & 51 & 323 & 481 & 425 & 1285 \\
\hline Safaeian M (ATBC $\left.{ }^{(\mathrm{e})}\right)$ & 1 & 11 & 25 & 13 & 61 & 51 & 374 & 844 & 476 & 2062 \\
\hline Safaeian M $\left(\mathrm{AHS}^{(\mathrm{f})}\right)$ & 2 & 6 & 10 & 10 & 26 & 1 & 8 & 26 & 10 & 60 \\
\hline Schoemaker M J(Denmark) & 1 & 38 & 83 & 40 & 204 & 8 & 56 & 83 & 72 & 222 \\
\hline Schoemaker M J(Finland) & 4 & 22 & 69 & 30 & 160 & 3 & 30 & 63 & 36 & 156 \\
\hline Shete (England) & 26 & 179 & 426 & 231 & 1031 & 82 & 533 & 818 & 697 & 2169 \\
\hline Shete (America) & 46 & 405 & 796 & 497 & 1997 & 123 & 785 & 1327 & 1031 & 3439 \\
\hline Shete (France) & 34 & 386 & 912 & 454 & 2210 & 59 & 508 & 978 & 626 & 2464 \\
\hline Shete (German) & 16 & 147 & 336 & 179 & 819 & 28 & 177 & 352 & 233 & 881 \\
\hline Shete (Sweden) & 20 & 195 & 430 & 235 & 1055 & 54 & 264 & 456 & 372 & 1176 \\
\hline
\end{tabular}

(b): National Cancer Institute glioma case-control study. (c): National Institute of Occupational Safety and Health glioma case-control study. (d): glioma cases and controls identified from the Prostate, Lung, Colorectal and Ovarian Cancer Screening Trial. (e): glioma cases and controls identified from the Alpha-Tocopherol, Beta-Carotene Cancer Prevention Study. (f): glioma cases and controls identified from the Agricultural Health Study

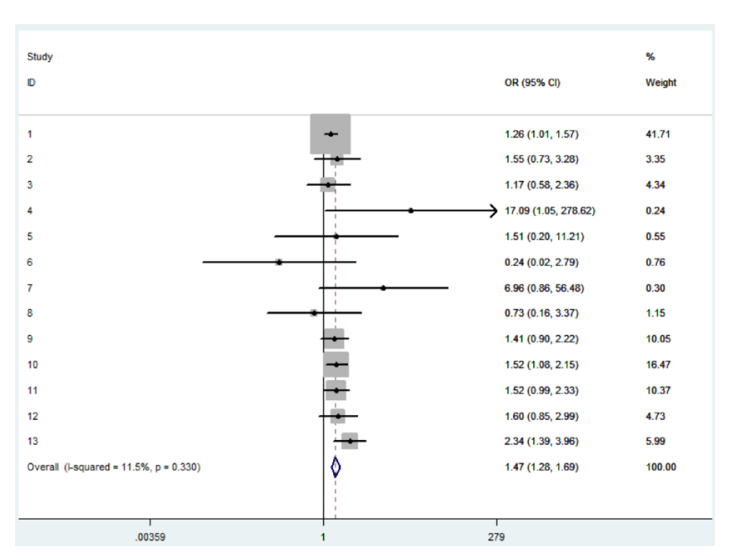

Figure 2. Meta-Analysis for the Association between the rs6010620 Polymorphism in RTEL1 gene and the Risk of Glioma: Total Analysis (GG+ AG vs. AA)

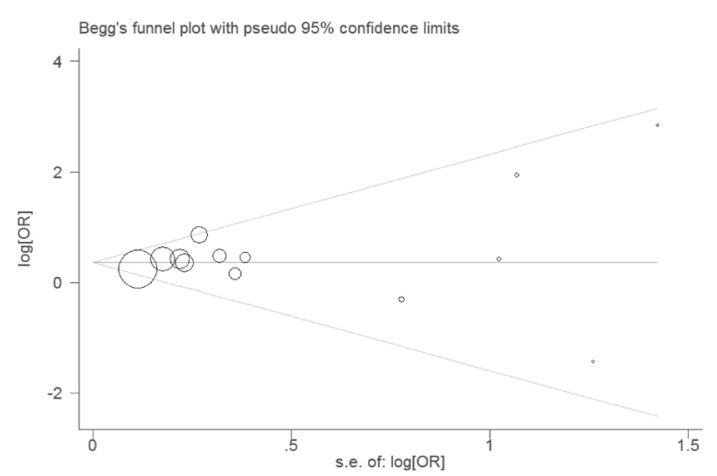

Figure 3. Funnel Plot For Evaluation of Publication Bias in the Selection of Studies on the Association between the rs6010620 Polymorphism in RTEL1 gene and the risk of Glioma (GG+ AG vs. AA)

Table 3. Summary of Total Pooled Results from Different Comparative Genetic Models

\begin{tabular}{llllllc}
\hline Genetic models & OR $(95 \% \mathrm{CI})$ & $\mathrm{Z}^{(\mathrm{g})}$ & $p$ & $\mathrm{I}^{2(\mathrm{~h})}(\%)$ & $\begin{array}{c}\text { Effect } \\
\text { models }\end{array}$ \\
\hline GG+AG vs. AA & $1.474(1.282-1.694)$ & 5.46 & $p<0.001$ & 11.5 & $\mathrm{~F}^{(\mathrm{i})}$ \\
GG $v s$. AG+AA & $1.368(1.194-1.566)$ & 4.52 & $p<0.001$ & 63 & $\mathrm{R}^{(\mathrm{j})}$ \\
GG $v$ s. AA & $1.819(1.539-2.150)$ & 7.01 & $p<0.001$ & 15.5 & $\mathrm{~F}$ \\
AG $v$ s. AA & $1.284(1.110-1.485)$ & 3.36 & $\mathrm{P}<0.001$ & 0 & $\mathrm{~F}$ \\
G vs. A & $1.362(1.174-1.579)$ & 4.08 & $p<0.001$ & 85 & $\mathrm{R}$
\end{tabular}

(g): Test for overall effect. (h): Index of heterogeneity. (i): Fixed-effects mode. (j): Random-effect model investigated the possible publication bias by using the funnel plot and Begg's linear regression test.

\section{Results}

Study characteristics

A total of 120 results were obtained after first search in Pubmed, Embase, CNKI, Wanfang, and Weipu databases. 100 articles were eliminated after checking the titles and abstracts. Further selecting these articles, 14 of them were rejected because 6 of them were not relevant to target gene polymorphisms, three of them were not the case-control design, two of them didn't have sufficient data, and three of them contained repeated or overlapped data. Thus, a total of 6 articles were identified. Further screening, one of them was excluded because it didn't meet the HWE in the control groups. Finally, 14 case-control studies from five articles met the inclusion criteria ( Shete et al., 2009; Schoemaker et al., 2010; Li et al., 2013a; Safaeian et al., 2013; Walsh et al., 2013), shown in Figure 1. Summary of the properties of studies is listed in Table1. The genotype and allele distributions are listed in Table 2.

\section{Quantitative synthesis}

The association between the rs 6010620 polymorphism in RTEL1 gene and risk of glioma was analyzed in a total of 8, 292 cases and 12, 419 controls from 14 case-control studies. We performed heterogeneity in the dominant model (GG + AG vs. AA), and the results indicated low heterogeneity $\left(\chi^{2}=13.56, \mathrm{I}^{2}=11.5 \%, p<0.001\right)$. Hence, we pooled the 14 studies using the fixed-effects model to analysis the association. By total analysis, significant association between rs6010620 polymorphism in RTEL1 gene and risk of glioma were found in the dominant model $(\mathrm{GG}+\mathrm{AG} v s$. $\mathrm{AA}: \mathrm{OR}=1.474,95 \% \mathrm{CI}=1.282$ $1.694, \mathrm{I}^{2}=11.5 \%$ ) (Figure2) and other comparison models. The total pooled results are summarized in Table 3. No publication bias was checked in both the funnel plot and the Begg's linear regression test $(\mathrm{t}=0.97, p=0.352)$ (Figure 3)

In the subgroup analysis by ethnicity, statistically significant associations were obtained both in Caucasian $(\mathrm{OR}=1.627,95 \% \mathrm{CI}=1.358-1.950, p<0.01)$ and Asian 
Table 4. Summary of Different Comparative Results

\begin{tabular}{|c|c|c|c|c|c|c|c|c|c|c|}
\hline \multirow[t]{2}{*}{ rs6010620 } & \multicolumn{2}{|c|}{ GG+AG vs. AA } & \multicolumn{2}{|c|}{ GG vs. AG+AA } & \multicolumn{2}{|c|}{ GG vs. AA } & \multicolumn{2}{|c|}{ GA vs. AA } & \multicolumn{2}{|l|}{ G vs. A } \\
\hline & OR $(95 \%$ CI $)$ & $p$ & OR (95 \% CI) & $p$ & OR (95 \% CI) & $p$ & OR (95 \% CI) & $p$ & OR $(95 \%$ CI $)$ & $p$ \\
\hline $\begin{array}{l}\text { total } \\
\text { subgroup by }\end{array}$ & $\begin{array}{l}1.474(1.282-1.694) \\
\text { py ethnicity }\end{array}$ & $p<0.001$ & $1.368(1.194-1.566)$ & $p<0.001$ & $1.819(1.539-2.150)$ & $p<0.001$ & $1.284(1.110-1.485)$ & $p<0.001$ & $1.362(1.174-1.579)$ & $p<0.001$ \\
\hline Asian & $1.259(1.010-1.569)$ & 0.04 & $2.044(1.369-2.052)$ & $p<0.001$ & $2.157(1.425-3.264)$ & $p<0.001$ & $1.124(0.892-1.417)$ & & $6(1.110-1.560)$ & 0.02 \\
\hline Caucasian & $1.627(1.358-1.950)$ & $p<0.001$ & $1.329(1.163-1.519)$ & $p<0.001$ & $1.762(1.468-2.115)$ & $p<0.001$ & $1.396(1.156-1.686)$ & 0.001 & $1.364(1.160-1.605)$ & $p<0.001$ \\
\hline
\end{tabular}

$(\mathrm{OR}=1.259,95 \% \mathrm{CI}=1.010-1.569, p=0.04)$. Summary of different comparative results for subgroup analyses is listed in Table 4.

\section{Discussion}

Glioma are the most common adults tumors of the central brain, have high mortality and morbidity (Ahmed et al., 2014). However, the mechanism of glioma was unclear. Up to date previous published articles found that many gene variations may associate with glioma risk, such as X-ray repair complementing group 1 gene (XRCC1) mutations, X-ray repair complementing group 3 gene (XRCC3) mutations, isocitrate dehydrogenase gene (IDH) mutations etc. The gene polymorphisms increased or decreased glioma sensibility by regulating the proliferation and apoptosis of cells. (Das et al., 2013; Li et al., 2013b) They revealed that gene mutations may contribute to the occurrence of glioma. Previous study found that mutations of RTEL1 gene may result in shortened telomere length, destroyed chromosome, and translocations, which revealed the possibility that RTEL1 protein contribute to protect the genome against instability (Ding et al., 2004; Barber et al., 2008; Uringa et al., 2012). One of the important candidate genetic variants is rs6010620 of RTEL1 gene which located on chromosome 20q13.33 and has been widely reported. Previous studies have revealed that gene mutation of rs6010620 may lead to glioma. However, the results were inconclusive. Thus, we conducted the current comprehensive metaanalysis to assess the association between the rs6010620 polymorphism in RTEL1 gene and risk of glioma.

Our study showed that the rs6010620 polymorphism in RTEL1 gene may increase risk of glioma $(\mathrm{OR}=1.474$, $95 \% \mathrm{CI}=1.282-1.694, p<0.001$ for $\mathrm{GG}+\mathrm{AG} v s$. AA) than the controls. It indicated that the person who carried the $\mathrm{G}$ allele will increase $47 \%$ risk of glioma. Due to different ethnicity have different frequencies of alleles. Therefore, we performed subgroup analysis according to ethnicity, which can decrease biases. In subgroup analysis, we found that rs6010620 polymorphism in RTEL1 gene increased risk of glioma in Caucasian $(\mathrm{OR}=1.627,95 \% \mathrm{CI}=1.358$ $1.950, p<0.001)$, as well as among Asian $(\mathrm{OR}=1.259$, 95\% CI=1.010-1.569, $p=0.04)$. However, we must be careful when refer to the results. Because only one article concerning Asian was included, so the results may have insufficient power to reveal a reliable association. In future, it is need to perform larger sample size studies to confirm the results both in Asian and in other ethnic groups.

Compared with the previous meta-analysis reported by Zhao W et al. (Zhao et al., 2014), our study including a total of 8, 292 cases and 12, 419 controls from 14 casecontrol studies controls, which has a larger sample size than the previous meta-analysis reported by Zhao $\mathrm{W}$ et al. (Zhao et al., 2014). In addition, there is a error in it that genotype frequencies of control group are not consistent with HWE in one included article (Chen et al., 2011). It is a serious mistake may result in bias, so the results of previous study may have limited power to reveal a reliable association. Thus, our study provided more reliable association between the rs6010620 polymorphism in RTEL1 gene and risk of glioma which resulted in more reliable conclusion. In addition, previous studies have found the association between the gene polymorphisms and glioma risk, such as the Glutathione S-transferases (GSTs) gene polymorphism (Sima et al., 2012). It may contribute to the occurrence of glioma. However, compare with our meta-analysis, it may have limited power to reveal a reliable association with a small sample. So, larger sample is needed to confirm the results in the future.

Several limitations in this meta-analysis should be mentioned. First of all, more accurate OR should adjusted be for age, sex, drinking, smoking, and other factors that are associated with cancer risk (Zhu et al., 2013). Second, in subgroup analysis by ethnicities, because all studies were from Asian and Caucasian, so the pooled results may be applicable to these two ethnic groups. Third, interactions of gene-gene and gene-environment interaction were not discussed, due to lack of original information (Li et al., 2012). Despite of these limitations mentioned above, we tried our best to minimize the bias through the whole process by identification, data selection, and statistical analysis as well as in the control of publication bias and sensitivity. By this way, the reliability of the results is ensured.

In conclusion, our study suggested that the rs6010620 polymorphism in RTEL1 may increase risk of glioma. However well-designed case-control studies with lager sample size focusing on more ethnicities or glioma type are needed to validate our findings in future.

\section{References}

Adelman CA, Boulton SJ (2010). Metabolism of postsynaptic recombination intermediates. FEBS letters, 584, 3709-16.

Ahmed R, Oborski MJ, Hwang M, Lieberman FS, Mountz JM (2014). Malignant gliomas: current perspectives in diagnosis, treatment, and early response assessment using advanced quantitative imaging methods. Cancer Manag Res, 6, 149-70

Bai C, Connolly B, Metzker ML, et al (2000). Overexpression of M68/DcR3 in human gastrointestinal tract tumors independent of gene amplification and its location in a fourgene cluster. Proc Natl Acad Sci USA, 97, 1230-5.

Barber LJ, Youds JL, Ward JD, et al (2008). RTEL1 maintains genomic stability by suppressing homologous recombination. Cell, 135, 261-71.

Blackburn EH, Greider CW, Szostak JW (2006). Telomeres and telomerase: the path from maize, Tetrahymena and yeast to human cancer and aging. Nat Med, 12, 1133-8. 
Bondy ML, Scheurer ME, Malmer B, et al (2008). Brain tumor epidemiology: consensus from the Brain Tumor Epidemiology Consortium. Cancer, 113, 1953-68.

Chen H, Chen Y, Zhao Y, et al (2011). Association of sequence variants on chromosomes 20,11, and 5 (20q13. 33, 11q23. 3 , and $5 \mathrm{p} 15.33$ ) with glioma susceptibility in a Chinese population. Am J Epidemiol, 173, 915-22.

Das BR, Tangri R, Ahmad F, Roy A, Patole K (2013). Molecular investigation of isocitrate dehydrogenase gene (IDH) mutations in gliomas: first report of IDH2 mutations in Indian patients. Asian Pac J Cancer Prev, 14, 7261-4.

Ding H, Schertzer M, Wu X, et al (2004). Regulation of Murine Telomere Length by $<\mathrm{i}>\mathrm{Rtel}<\mathrm{i}>$ : An Essential Gene Encoding a Helicase-like Protein. Cell, 117, 873-86.

Dolecek TA, Propp JM, Stroup NE, Kruchko C (2012). CBTRUS statistical report: primary brain and central nervous system tumors diagnosed in the United States in 2005-2009. Neuro Oncol, 14, 1-49.

Hackett JA, Greider CW (2002). Balancing instability: dual roles for telomerase and telomere dysfunction in tumorigenesis. Oncogene, 21, 619-26

Li G, Jin T, Liang H, et al (2013a). RTEL1 tagging SNPs and haplotypes were associated with glioma development. Diagn Pathol, 8, 83.

Li L, Tian J, Tian H, et al (2012). Quality and transparency of overviews of systematic reviews. J Evid Based Med, 5, 166-73.

Li-RLL, Li S, Qin X (2013b). Association of XRCC3 Thr241Met polymorphisms and gliomas risk: evidence from a metaanalysis. Asian Pac J Cancer Prev, 14, 4243-7.

Muleris M, Almeida A, Gerbault-Seureau M, Malfoy B, Dutrillaux B (1995). Identification of amplified DNA sequences in breast cancer and their organization within homogeneously staining regions. Genes Chromosomes Cancer, 14, 155-63.

Pitti RM, Marsters SA, Lawrence DA, et al (1998). Genomic amplification of a decoy receptor for Fas ligand in lung and colon cancer. Nature, 396, 699-703.

Safaeian M, Rajaraman P, Hartge P, et al (2013). Joint effects between five identified risk variants, allergy, and autoimmune conditions on glioma risk. Cancer Causes Control, 24, 1885-91.

Schoemaker MJ, Robertson L, Wigertz A, et al (2010). Interaction between 5 genetic variants and allergy in glioma risk. Am J Epidemiol, 171, 1165-73.

Shete S, Hosking FJ, Robertson LB, et al (2009). Genome-wide association study identifies five susceptibility loci for glioma. Nat Genet, 41, 899-904.

Sima XT, Zhong WY, Liu JG, You C (2012). Lack of association between GSTM1 and GSTT1 polymorphisms and brain tumour risk. Asian Pac J Cancer Prev, 13, 325-8.

Uringa EJ, Lisaingo K, Pickett HA, et al (2012). RTEL1 contributes to DNA replication and repair and telomere maintenance. Mol Biol Cell, 23, 2782-92

Uringa EJ, Youds JL, Lisaingo K, Lansdorp PM, Boulton SJ (2011).RTEL1: an essential helicase for telomere maintenance and the regulation of homologous recombination. Nucleic Acids Res, 39, 1647-55.

Vannier JB, Sarek G, Boulton SJ, (2014). RTEL1: Functions of a disease-associated helicase. Trends Cell Biol, 24, 416-25

Walsh KM, Rice T, Decker PA, et al (2013). Genetic variants in telomerase-related genes are associated with an older age at diagnosis in glioma patients: evidence for distinct pathways of gliomagenesis. Neuro Oncol, 15, 1041-7.

Wu X, Sandhu S, Nabi Z, Ding H (2012). Generation of a mouse model for studying the role of upregulated RTEL1 activity in tumorigenesis. Transgenic Res, 21, 1109-15.
Youds JL, Mets DG, McIlwraith MJ, et al (2010). RTEL-1 enforces meiotic crossover interference and homeostasis. Science, 327, 1254-8.

Zhao W, Bian Y, Zhu W, Zou P, Tang G (2014). Regulator of telomere elongation helicase 1 (RTEL1) rs6010620 polymorphism contribute to increased risk of glioma. Tumor Biol, 35, 5259-66.

Zhu LX, Ho SC, Wong TK (2013). Effectiveness of health education programs on exercise behavior among patients with heart disease: a systematic review and meta-analysis. J Evid Based Med, 6, 265-301. 